\title{
Uniform Approximate Estimation for Nonlinear Nonhomogenous Stochastic System with Unknown Parameter
}

\author{
Xiu Kan and Huisheng Shu \\ School of Information Science and Technology, Donghua University, Shanghai 200051, China \\ Correspondence should be addressed to Huisheng Shu, hsshu@dhu.edu.cn
}

Received 21 June 2012; Accepted 3 August 2012

Academic Editor: Jun Hu

Copyright (C) 2012 X. Kan and H. Shu. This is an open access article distributed under the Creative Commons Attribution License, which permits unrestricted use, distribution, and reproduction in any medium, provided the original work is properly cited.

The error bound in probability between the approximate maximum likelihood estimator (AMLE) and the continuous maximum likelihood estimator (MLE) is investigated for nonlinear nonhomogenous stochastic system with unknown parameter. The rates of convergence of the approximations for Itô and ordinary integral are introduced under some regular assumptions. Based on these results, the in probability rate of convergence of the approximate loglikelihood function to the true continuous log-likelihood function is studied for the nonlinear nonhomogenous stochastic system involving unknown parameter. Finally, the main result which gives the error bound in probability between the ALME and the continuous MLE is established.

\section{Introduction}

It is now well known that the parameter estimation is one of the foundational problems in stochastic differential equations which are used to model practical systems that with random influences. Since 1962, Arato et al. who first applied parameter estimation to a geophysical problem in [1]. Various parameter estimation methods have been developed for many advanced models with an increasing number of application to physical, biological and financial systems. Over the past few decades, a lot of effective approaches have proposed in this research area, see for example, [2-5]. In particular, maximum likelihood estimation (MLE) gives a unified approach to estimation, which is well defined in the case of the normal distribution and many other statistical models. Therefore the MLE technique has been widely used for the parameter estimation problem of stochastic systems [6]. Byes estimation (BE), which is a decision rule that minimizes the posterior expected value of a loss function, has been developed in [7]. Since some inconvenience is encountered in the 
real-time application that location and scale parameters are not uniquely determined, Mestimator has been studied toward the theory of robust estimation [8]. Other widely used parameter estimation methods can be generally categorized as least squares estimation (LSE), maximum probability estimation (MPE), minimum distance estimation (MDE), minimum contrast estimation (MCE), and filtering method for parameter estimation, see for example, [9-18] and the references therein.

In reality, nonhomogenous stochastic differential equations are useful for modeling term structure of interest rates in finance and other fields. A large number of results have been published in the literature on a variety of research topics including strong or weak consistency and asymptotic efficiency as well as asymptotic normality on various parameter estimators of nonhomogenous stochastic systems $[19,20]$. On the other hand, recognizing that nonlinearity is commonly encountered in engineering practice, the parameter estimation problem for nonlinear nonhomogenous stochastic systems deserves more research attention from both the theoretical and practical viewpoints and, accordingly, some promising results have been reported. For example, weak consistency, asymptotic normality, and convergence of moments of MLE and BE of the drift parameter in the nonlinear nonhomogenous Itô stochastic differential equations having nonstationary solutions have been studied in [21] for the small noise asymptotic case. In [22], the martingale approach but under some stronger regularity conditions has been used to study strong consistency and asymptotic normality for nonlinear nonhomogenous stochastic system in the large sample case. It should be pointed out that, so far, many parameter estimation methods and corresponding probability properties have been widely investigated for nonlinear nonhomogenous Itô stochastic differential equation with constant diffusion. Unfortunately, the parameter problem of general nonlinear nonhomogenous system has gained much less research attention despite its potential in practical application.

The stochastic processes which can be observed continuously over a specified time period are first used to model real system for the most part [23, 24]. In practice, it is obviously impossible to observe a process continuously over any given time period, due to the limitations on the precision of the measuring instrument or to unavailability of observations at every time point, and so forth. In other words, stochastic inference based on discrete observations is of major importance in dealing with practical problems. Hence, parameter estimation problem based on discrete observations has naturally become a hot topic in recent years $[25,26]$. An approximation method has been proposed based on the discretization of the continuous time likelihood function in [27] for linear stochastic differential equation. A numerical approximate likelihood method has been developed in [28] based on iterations of the Gaussian transition densities emanating from the Euler scheme. [29] has used a specific transformation of the diffusion to obtain accurate theoretical approximations based on the Hermite function expansions and studied the asymptotic behavior of the approximate MLE. Up to now, although some parameter estimation problems have been established based on discretization scheme, how close are the discrete parameter estimator to the true continuous one for general nonlinear nonhomogenous stochastic system has not been fully studied due probably to the mathematical complexity, and this situation motivates our present paper.

Summarizing the above discussions, in this paper, we are motivated to study the rate of convergence of the approximate maximum likelihood estimator (AMLE) to the true continuous MLE for a class of general nonlinear nonhomogenous stochastic system with unknown parameter. The main contributions of this paper lie in the following aspects. (1) The Ito type approximation for the stochastic integral is introduced to obtain an approximate loglikelihood function. (2) The rate of convergence of the approximation is investigated for Itô type 
integral. (3) The in probability rate of convergence of the approximate log-likelihood function is established for the nonlinear nonhomogenous stochastic system involving unknown parameter. (4) The error bound in probability of the ALME and the LME is studied for the nonlinear nonhomogenous stochastic system. The rest of this paper is outlined as follows. In Section 2, the approximate log-likelihood function is proposed and the problem under consideration is formulated. In Section 3, several lemmas are given to analyze the rates of convergence of the approximations for Itô and ordinary integral; furthermore, the main results are discussed to analyze the rate of convergence of the approximate log-likelihood function and the error bound of the ALME and the LME. Finally, we conclude the paper in Section 4.

\section{Problem Formulation and Preliminaries}

Consider the real valued diffusion process $X_{t}, t \geq 0$ on $\left(\Omega, \mathcal{F},\left\{\mathcal{F}_{t}\right\}_{t \geq 0}, \mathbf{P}\right)$ satisfying the following stochastic differential equation:

$$
d X_{t}=\theta f\left(t, X_{t}\right) d t+g\left(t, X_{t}\right) d W_{t}
$$

where $W_{t}, t \geq 0$ is a standard Wiener process adapted to $\mathcal{F}_{t}, t \geq 0$ such that for $0 \leq s<t$, $W_{t}-W_{s}$ is independent of $\mathcal{F}_{s}, \theta \in \Theta$ open in $\mathbb{R}$ is the unknown parameter to be estimated. Let $\theta_{0}$ be the true value of the parameter $\theta$.

Throughout this paper $C$ is a generic constant, we use following notations:

$$
f_{x}=\frac{\partial f}{\partial x}, \quad f_{t}=\frac{\partial f}{\partial t}, \quad f_{x x}=\frac{\partial^{2} f}{\partial x^{2}}, \quad f_{t t}=\frac{\partial^{2} f}{\partial t^{2}}, \quad f_{t x}=\frac{\partial^{2} f}{\partial t \partial x} .
$$

We assume the following condition:

(A1) $f(\cdot, \cdot)$ and $g(\cdot, \cdot)$ are Lipschitz continuous in $X_{t} \in \mathbb{R}$ uniformly in $t \in \mathbb{R}_{+}$, that is, there exists a constant $K \geq 0$ such that

$$
\left|f\left(t, X_{1}\right)-f\left(t, X_{2}\right)\right|^{2} \vee\left|g\left(t, X_{1}\right)-g\left(t, X_{2}\right)\right|^{2} \leq K\left|X_{1}-X_{2}\right|^{2},
$$

for any $t \in \mathbb{R}_{+}$and $X_{1}, X_{2} \in \mathbb{R}$.

(A2) $f(\cdot, \cdot)$ and $g(\cdot, \cdot)$ satisfy linear growth condition, that is, there exists a constant $K \geq 0$ such that

$$
|f(t, x)|^{2} \vee|g(t, x)|^{2} \leq K\left(1+|x|^{2}\right),
$$

for any $t \in \mathbb{R}_{+}$and $x \in \mathbb{R}$.

(A3)

$$
\inf _{\left\{t, X_{t}\right\}} g^{2}\left(t, X_{t}\right)>0 .
$$


(A4)

$$
\forall p \geq 0, \quad \sup _{\left\{t, X_{t}\right\}} \mathbb{E}\left|f\left(t, X_{t}\right)\right|^{p}<\infty, \quad \sup _{\left\{t, X_{t}\right\}} \mathbb{E}\left|g\left(t, X_{t}\right)\right|^{p}<\infty
$$

(A5) $j f(\cdot, \cdot)$ and $g(\cdot, \cdot)$ are continuously differentiable with respect to $X_{t}$ up to order $j \geq 1$ and

$$
\begin{array}{ll}
\sup _{0 \leq t \leq T} \mathbb{E}\left|f_{x}\left(t, X_{t}\right)\right|^{8}<\infty, & \sup _{0 \leq t \leq T} \mathbb{E}\left|g_{x}\left(t, X_{t}\right)\right|^{16}<\infty, \\
\sup _{0 \leq t \leq T} \mathbb{E}\left|f_{x x}\left(t, X_{t}\right)\right|^{8}<\infty, & \sup _{0 \leq t \leq T} \mathbb{E}\left|g_{x x}\left(t, X_{t}\right)\right|^{16}<\infty .
\end{array}
$$

$(\mathrm{A} 6)_{k} f(\cdot, \cdot)$ and $g(\cdot, \cdot)$ are continuously differentiable with respect to $t$ up to order $k \geq 1$ and

$$
\begin{array}{ll}
\sup _{0 \leq t \leq T} \mathbb{E}\left|f_{t}\left(t, X_{t}\right)\right|^{4}<\infty, & \sup _{0 \leq t \leq T} \mathbb{E}\left|g_{t}\left(t, X_{t}\right)\right|^{8}<\infty, \\
\sup _{0 \leq t \leq T} \mathbb{E}\left|f_{t t}\left(t, X_{t}\right)\right|^{4}<\infty, & \sup _{0 \leq t \leq T} \mathbb{E}\left|g_{t t}\left(t, X_{t}\right)\right|^{4}<\infty .
\end{array}
$$

(A7)

$$
\sup _{0 \leq t \leq T} \mathbb{E}\left|f_{t x}\left(t, X_{t}\right)\right|^{8}<\infty, \quad \sup _{0 \leq t \leq T} \mathbb{E}\left|g_{t x}\left(t, X_{t}\right)\right|^{8}<\infty
$$

$$
\mathbb{E}\left|X_{0}\right|^{8}<\infty
$$

Remark 2.1. As (A1) and (A2) are established, it is well known that stochastic differential equation (2.1) has a unique solution. Please see the details in [30].

Denote $X_{0}^{T}=\left\{X_{t}, 0 \leq t \leq T\right\}$. Let $P_{\theta}^{T}$ be the measure generated on the space $\left(C_{T}, B_{T}\right)$ of the continuous functions on $[0, T]$ with the associated Borel $\sigma$-algebra $B_{T}$ generated under the supremum norm by the process $X_{0}^{T}$ and $P_{0}^{T}$ be the standard Wiener measure. Under assumptions (A3) and (A4), the measure $P_{\theta}^{T}$ and $P_{0}^{T}$ are equivalent and the Randon-Nikodym derivative of $P_{\theta}^{T}$ with respect to $P_{0}^{T}$ is given by

$$
\frac{d P_{\theta}^{T}}{d P_{0}^{T}}=\exp \left\{\theta \int_{0}^{T} \frac{f\left(t, X_{t}\right)}{g^{2}\left(t, X_{t}\right)} d X_{t}-\frac{\theta^{2}}{2} \int_{0}^{T} \frac{f^{2}\left(t, X_{t}\right)}{g^{2}\left(t, X_{t}\right)} d t\right\}
$$


along the sample path $X_{0}^{T}$. Let

$$
L_{T}(\theta)=\log \frac{d P_{\theta}^{T}}{d P_{0}^{T}}=\theta \int_{0}^{T} \frac{f\left(t, X_{t}\right)}{g^{2}\left(t, X_{t}\right)} d X_{t}-\frac{\theta^{2}}{2} \int_{0}^{T} \frac{f^{2}\left(t, X_{t}\right)}{g^{2}\left(t, X_{t}\right)} d t
$$

be the log-likelihood function. The maximum likelihood estimate (MLE) of $\theta$ is defined as

$$
\theta_{T}=\arg \max _{\theta \in \Theta} L_{T}(\theta)\left\{\int_{0}^{T} \frac{f\left(t, X_{t}\right)}{g^{2}\left(t, X_{t}\right)} d X_{t}\right\}\left\{\int_{0}^{T} \frac{f^{2}\left(t, X_{t}\right)}{g^{2}\left(t, X_{t}\right)} d t\right\}^{-1}
$$

Now, we study the approximation of the MLE $\theta_{T}$ when stochastic $X_{t}$ is observed at the discrete-time points $0=t_{0}<t_{1}<\cdots<t_{n}=T$ with $t_{i}-i h, i=0,1,2, \ldots, n$ such that $h \rightarrow 0$ as $n \rightarrow \infty$. Itô approximation of the stochastic integral and rectangular approximation of the ordinary integral in the log-likelihood (2.12) yields the approximate log-likelihood function:

$$
L_{n, T}(\theta)=\theta\left\{\sum_{i=1}^{n} \frac{f\left(t_{i-1}, X_{t_{i-1}}\right)}{g^{2}\left(t_{i-1}, X_{t_{i-1}}\right)}\left(X_{t_{i}}-X_{t_{i-1}}\right)\right\}-\frac{\theta^{2}}{2}\left\{\sum_{i=1}^{n} \frac{f^{2}\left(t_{i-1}, X_{t_{i-1}}\right)}{g^{2}\left(t_{i-1}, X_{t_{i-1}}\right)}\left(t_{i}-t_{i-1}\right)\right\} .
$$

The corresponding approximate maximum likelihood estimator (AMLE) is established as follow:

$$
\theta_{n, T}=\left\{\sum_{i=1}^{n} \frac{f\left(t_{i-1}, X_{t_{i-1}}\right)}{g^{2}\left(t_{i-1}, X_{t_{i-1}}\right)}\left(X_{t_{i}}-X_{t_{i-1}}\right)\right\}\left\{\sum_{i=1}^{n} \frac{f^{2}\left(t_{i-1}, X_{t_{i-1}}\right)}{g^{2}\left(t_{i-1}, X_{t_{i-1}}\right)}\left(t_{i}-t_{i-1}\right)\right\}^{-1} .
$$

The main purpose of this paper is to study the rate of the convergence of the approximate log-likelihood functions and furthermore analyze the error bound in probability between the AMLE and the continuous MLE.

\section{Main Results}

Firstly, let us give the following lemmas which will be used in the proof of our main results.

Lemma 3.1. Under the assumptions $(A 1)-(A 4),(A 5)_{2}$, and $(A 6)_{1}$, one has

$$
\mathbb{E}\left|\sum_{i=1}^{n} \frac{f^{2}\left(t_{i-1}, X_{i-1}\right)}{g^{2}\left(t_{i-1}, X_{i-1}\right)}\left(t_{i}-t_{i-1}\right)-\int_{0}^{T} \frac{f^{2}\left(t, X_{t}\right)}{g^{2}\left(t, X_{t}\right)} d t\right|^{2} \leq C \frac{T^{3}}{n^{2}}
$$


Proof. By Itô formula we can derive that for $t \in[0, T]$,

$$
\begin{aligned}
& \frac{f^{2}\left(t, X_{t}\right)}{g^{2}\left(t, X_{t}\right)}-\frac{f^{2}\left(t_{i-1}, X_{i-1}\right)}{g^{2}\left(t_{i-1}, X_{i-1}\right)} \\
& \quad \int_{t_{i-1}}^{t} \frac{2 f\left(u, X_{u}\right) f_{u}\left(u, X_{u}\right) g^{2}\left(u, X_{u}\right)-2 f^{2}\left(u, X_{u}\right) g\left(u, X_{u}\right) g_{u}\left(u, X_{u}\right)}{g^{4}\left(u, X_{u}\right)} d u \\
& \quad+\int_{t_{i-1}}^{t} \frac{2 f\left(u, X_{u}\right) f_{x}\left(u, X_{u}\right) g^{2}\left(u, X_{u}\right)-2 f^{2}\left(u, X_{u}\right) g\left(u, X_{u}\right) g_{x}\left(u, X_{u}\right)}{g^{4}\left(u, X_{u}\right)} \theta f\left(u, X_{u}\right) d u \\
& \quad+\int_{t_{i-1}}^{t}\left[f_{x}^{2}\left(u, X_{u}\right) g^{2}\left(u, X_{u}\right)+3 f^{2}\left(u, X_{u}\right) g_{x}^{2}\left(u, X_{u}\right)-4 f\left(u, X_{u}\right) g\left(u, X_{u}\right) f_{x}\left(u, X_{u}\right) g_{x}\left(u, X_{u}\right)\right] d u \\
& \quad+\int_{t_{i-1}}^{t}\left[f\left(u, X_{u}\right) g^{2}\left(u, X_{u}\right) f_{x x}\left(u, X_{u}\right)-f^{2}\left(u, X_{u}\right) g\left(u, X_{u}\right) g_{x x}\left(u, X_{u}\right)\right] d u \\
& \quad+\int_{t_{i-1}}^{t} \frac{2 f\left(u, X_{u}\right) f_{x}\left(u, X_{u}\right) g^{2}\left(u, X_{u}\right)-2 f^{2}\left(u, X_{u}\right) g\left(u, X_{u}\right) g_{x}\left(u, X_{u}\right)}{g^{4}\left(u, X_{u}\right)} d W_{u} \\
& \triangleq \int_{t_{i-1}}^{t} F_{1}\left(u, X_{u}\right) d u+\int_{t_{i-1}}^{t} F_{2}\left(u, X_{u}\right) d W_{u \prime}
\end{aligned}
$$

where

$$
\begin{aligned}
& F_{1}\left(u, X_{u}\right)=F_{11}\left(u, X_{u}\right)+F_{12}\left(u, X_{u}\right)+F_{13}\left(u, X_{u}\right)+F_{14}\left(u, X_{u}\right), \\
& F_{11}\left(u, X_{u}\right)=\frac{2 f\left(u, X_{u}\right) f_{u}\left(u, X_{u}\right) g^{2}\left(u, X_{u}\right)-2 f^{2}\left(u, X_{u}\right) g\left(u, X_{u}\right) g_{u}\left(u, X_{u}\right)}{g^{4}\left(u, X_{u}\right)}, \\
& F_{12}\left(u, X_{u}\right)=\frac{2 f\left(u, X_{u}\right) f_{x}\left(u, X_{u}\right) g^{2}\left(u, X_{u}\right)-2 f^{2}\left(u, X_{u}\right) g\left(u, X_{u}\right) g_{x}\left(u, X_{u}\right)}{g^{4}\left(u, X_{u}\right)} \theta f\left(u, X_{u}\right), \\
& F_{13}\left(u, X_{u}\right)=f_{x}^{2}\left(u, X_{u}\right) g^{2}\left(u, X_{u}\right)+3 f^{2}\left(u, X_{u}\right) g_{x}^{2}\left(u, X_{u}\right)-4 f\left(u, X_{u}\right) g\left(u, X_{u}\right) f_{x}\left(u, X_{u}\right) g_{x}\left(u, X_{u}\right), \\
& F_{14}\left(u, X_{u}\right)=f\left(u, X_{u}\right) g^{2}\left(u, X_{u}\right) f_{x x}\left(u, X_{u}\right)-f^{2}\left(u, X_{u}\right) g\left(u, X_{u}\right) g_{x x}\left(u, X_{u}\right) .
\end{aligned}
$$


For $F_{11}\left(u, X_{u}\right)$ and $F_{13}\left(u, X_{u}\right)$, by assumption (A3), (A4), (A5) $2,(\mathrm{~A} 6)_{1}$, and Hölder's inequality, one has

$$
\begin{aligned}
& \mathbb{E}\left(\int_{t_{i-1}}^{t} F_{11}\left(u, X_{u}\right)\right)^{2} \\
& \quad=\mathbb{E}\left(\int_{t_{i-1}}^{t} \frac{2 f\left(u, X_{u}\right) f_{u}\left(u, X_{u}\right) g^{2}\left(u, X_{u}\right)-2 f^{2}\left(u, X_{u}\right) g\left(u, X_{u}\right) g_{u}\left(u, X_{u}\right)}{g^{4}\left(u, X_{u}\right)} d u\right)^{2} \\
& \quad \leq C \mathbb{E} \int_{t_{i-1}}^{t} f^{2}\left(u, X_{u}\right) d u+C \mathbb{E} \int_{t_{i-1}}^{t} f_{u}^{2}\left(u, X_{u}\right) d u+C \mathbb{E} \int_{t_{i-1}}^{t} g_{u}^{2}\left(u, X_{u}\right) d u \\
& \left.\quad \leq C, \quad \int_{t_{i-1}}^{t} F_{13}\left(u, X_{u}\right)\right)^{2} \\
& \quad=\mathbb{E}\left(\int_{t_{i-1}}^{t} f_{x}^{2}\left(u, X_{u}\right) g^{2}\left(u, X_{u}\right)+3 f^{2}\left(u, X_{u}\right) g_{x}^{2}\left(u, X_{u}\right)\right. \\
& \left.\quad-4 f\left(u, X_{u}\right) g\left(u, X_{u}\right) f_{x}\left(u, X_{u}\right) g_{x}\left(u, X_{u}\right) d u\right)^{2} \\
& \quad \leq C \mathbb{E} \int_{t_{i-1}}^{t} f^{4}\left(u, X_{u}\right) d u+C \mathbb{E} \int_{t_{i-1}}^{t} g^{4}\left(u, X_{u}\right) d u+C \mathbb{E} \int_{t_{i-1}}^{t} f_{x}^{4}\left(u, X_{u}\right) d u+C \mathbb{E} \int_{t_{i-1}}^{t} g_{x}^{4}\left(u, X_{u}\right) d u \\
& \quad \\
& \quad \leq 4.4)
\end{aligned}
$$

Similarly, we have

$$
\mathbb{E}\left(\int_{t_{i-1}}^{t} F_{12}\left(u, X_{u}\right)\right)^{2} \leq C, \quad \mathbb{E}\left(\int_{t_{i-1}}^{t} F_{14}\left(u, X_{u}\right)\right)^{2} \leq C .
$$

This means

$$
\mathbb{E}\left(\int_{t_{i-1}}^{t} F 1\left(u, X_{u}\right)\right)^{2} \leq C
$$


Hence, it follows $C_{r}$ inequality that

$$
\begin{aligned}
\mathbb{E} \mid & \sum_{i=1}^{n} \frac{f^{2}\left(t_{i-1}, X_{i-1}\right)}{g^{2}\left(t_{i-1}, X_{i-1}\right)}\left(t_{i}-t_{i-1}\right)-\left.\int_{0}^{T} \frac{f^{2}\left(t, X_{t}\right)}{g^{2}\left(t, X_{t}\right)} d t\right|^{2} \\
& =\mathbb{E}\left|\sum_{i=1}^{n} \int_{t_{i-1}}^{t_{i}}\left[\frac{f^{2}\left(t_{i-1}, X_{i-1}\right)}{g^{2}\left(t_{i-1}, X_{i-1}\right)}\left(t_{i}-t_{i-1}\right)-\int_{0}^{T} \frac{f^{2}\left(t, X_{t}\right)}{g^{2}\left(t, X_{t}\right)}\right] d t\right|^{2} \\
& =\mathbb{E}\left|\sum_{i=1}^{n} \int_{t_{i-1}}^{t_{i}}\left[\int_{t_{i-1}}^{t} F_{1}\left(u, X_{u}\right) d u+\int_{t_{i-1}}^{t} F_{2}\left(u, X_{u}\right) d W_{u}\right] d t\right|^{2} \\
& \leq 2 \mathbb{E}\left|\sum_{i=1}^{n} \int_{t_{i-1}}^{t_{i}} \int_{t_{i-1}}^{t} F_{1}\left(u, X_{u}\right) d u d t\right|^{2}+2 \mathbb{E}\left|\sum_{i=1}^{n} \int_{t_{i-1}}^{t_{i}} \int_{t_{i-1}}^{t} F_{2}\left(u, X_{u}\right) d W_{u} d t\right|^{2} \triangleq 2 G_{1}+2 G_{2} .
\end{aligned}
$$

By assumptions (A3), (A4), (A5) 2 , and (A6) 1 , we obtain

$$
\begin{aligned}
G_{1}= & \mathbb{E}\left|\sum_{i=1}^{n} \int_{t_{i-1}}^{t_{i}} \int_{t_{i-1}}^{t} F_{1}\left(u, X_{u}\right) d u d t\right|^{2} \\
\leq & \mathbb{E} \sum_{i=1}^{n}\left(\int_{t_{i-1}}^{t_{i}} \int_{t_{i-1}}^{t} F_{1}\left(u, X_{u}\right) d u d t\right)^{2} \\
& +\mathbb{E} \sum_{1=i \neq j}^{n}\left(\int_{t_{i-1}}^{t_{i}} \int_{t_{i-1}}^{t} F_{1}\left(u, X_{u}\right) d u d t\right)\left(\int_{t_{j-1}}^{t_{j}} \int_{t_{j-1}}^{t} F_{1}\left(u, X_{u}\right) d u d t\right) \\
\leq & \mathbb{E} \sum_{i=1}^{n}\left(t_{i}-t_{i-1}\right) \int_{t_{i-1}}^{t_{i}}\left|\int_{t_{i-1}}^{t} F_{1}\left(u, X_{u}\right) d u d t\right|^{2} d t \\
& +\sum_{1=i \neq j}^{n}\left\{\mathbb{E}\left(\int_{t_{i-1}}^{t_{i}} \int_{t_{i-1}}^{t} F_{1}\left(u, X_{u}\right) d u d t\right)^{2} \mathbb{E}\left(\int_{t_{j-1}}^{t_{j}} \int_{t_{j-1}}^{t} F_{1}\left(u, X_{u}\right) d u d t\right)^{2}\right\}^{1 / 2} \\
\leq & \sum_{i=1}^{n}\left(t_{i}-t_{i-1}\right) \int_{t_{i-1}}^{t_{i}} \mathbb{E}\left|\int_{t_{i-1}}^{t} F_{1}\left(u, X_{u}\right) d u d t\right|^{2} d t \\
& +\sum_{1=i \neq j}^{n}\left\{\left(t_{i}-t_{i-1}\right) \int_{t_{i-1}}^{t_{i}} \mathbb{E}\left(\int_{t_{i-1}}^{t} F_{1}\left(u, X_{u}\right) d u\right)^{2} d t\left(t_{j}-t_{j-1}\right) \int_{t_{j-1}}^{t_{j}} \mathbb{E}\left(\int_{t_{j-1}}^{t} F_{1}\left(u, X_{u}\right) d u\right)^{2} d t\right\}^{1 / 2} \\
\leq & C \sum_{i=1}^{n}\left(t_{i}-t_{i-1}\right)^{3}+C \sum_{1=i \neq j}^{n}\left\{\left(t_{i}-t_{i-1}\right)^{3}\left(t_{j}-t_{j-1}\right)^{3}\right\}^{1 / 2} \leq C \frac{T^{3}}{n^{2}} .
\end{aligned}
$$


Due to the orthogonality, Itô isomorphism, the Cauchy-Schwarz inequality, assumption (A3), (A4), and (A5) 1 , we get

$$
\begin{aligned}
G_{2}= & \mathbb{E}\left|\sum_{i=1}^{n} \int_{t_{i-1}}^{t_{i}} \int_{t_{i-1}}^{t} F_{2}\left(u, X_{u}\right) d W_{u} d t\right|^{2} \\
\leq & \mathbb{E} \sum_{i=1}^{n}\left(\int_{t_{i-1}}^{t_{i}} \int_{t_{i-1}}^{t} F_{2}\left(u, X_{u}\right) d W_{u} d t\right)^{2} \\
& +\sum_{1=i \neq j}^{n} \mathbb{E}\left(\int_{t_{i-1}}^{t_{i}} \int_{t_{i-1}}^{t} F_{2}\left(u, X_{u}\right) d W_{u} d t\right)\left(\int_{t_{j-1}}^{t_{j}} \int_{t_{j-1}}^{t} F_{2}\left(u, X_{u}\right) d W_{u} d t\right) \\
\leq & \sum_{i=1}^{n}\left(t_{i}-t_{i-1}\right) \int_{t_{i-1}}^{t_{i}} \mathbb{E}\left|\int_{t_{i-1}}^{t} F_{2}\left(u, X_{u}\right) d W_{u} d t\right|^{2} d t \\
\leq & \sum_{i=1}^{n}\left(t_{i}-t_{i-1}\right) \int_{t_{i-1}}^{t_{i}} \int_{t_{i-1}}^{t} \mathbb{E}\left|F_{2}\left(u, X_{u}\right)\right|^{2} d u d t \\
\leq & C \sum_{i=1}^{n}\left(t_{i}-t_{i-1}\right) \int_{t_{i-1}}^{t_{i}}\left(t-t_{i-1}\right) d t \\
\leq & C \sum_{i=1}^{n}\left(t_{i}-t_{i-1}\right)^{3} \\
\leq & C \frac{T^{3}}{n^{2}} .
\end{aligned}
$$

Obviously, it follows from bounds for $G_{1}$ and $G_{2}$ that

$$
\mathbb{E}\left|\sum_{i=1}^{n} \frac{f^{2}\left(t_{i-1}, X_{i-1}\right)}{g^{2}\left(t_{i-1}, X_{i-1}\right)}\left(t_{i}-t_{i-1}\right)-\int_{0}^{T} \frac{f^{2}\left(t, X_{t}\right)}{g^{2}\left(t, X_{t}\right)} d t\right|^{2} \leq C \frac{T^{3}}{n^{2}}
$$

The proof is now complete.

Next, we will go on to analyze the rate of convergence of the approximations for Itô integral whose result will be used in the following theorems.

Lemma 3.2. Under the assumptions (A1)-(A4), $(A 5)_{2},(A 6)_{2},(A 7)$, and (A8), one has

$$
\mathbb{E}\left|\sum_{i=1}^{n} \frac{f\left(t_{i-1}, X_{t_{i-1}}\right)}{g\left(t_{i-1}, X_{t_{i-1}}\right)}\left(W_{t_{i}}-W_{t_{i-1}}\right)-\int_{0}^{T} \frac{f\left(t, X_{t}\right)}{g\left(t, X_{t}\right)} d W_{t}\right|^{2} \leq C \frac{T^{3}}{n^{2}}
$$


Proof. Let $\pi_{n}$ be the partition $\pi_{n}=0=t_{0}<t_{1}<\cdots<t_{n}=T, t_{i}=i h, i=0,1, \ldots, n$ such that $h \rightarrow 0$. Define $S$ and $S_{n}$ as

$$
\begin{gathered}
S=\int_{0}^{T} \frac{f^{2}\left(t, X_{t}\right)}{g^{2}\left(t, X_{t}\right)} d W_{t} \\
S_{n}=\sum_{i=1}^{n} \frac{f\left(t_{i-1}, X_{t_{i-1}}\right)}{g\left(t_{i-1}, X_{t_{i-1}}\right)}\left(W_{t_{i}}-W_{t_{i-1}}\right) .
\end{gathered}
$$

Let $\pi_{n}^{\prime}$ be a partition which is finer than $\pi_{n}$, obtained by choosing the mid point $\widehat{t}_{i-1}$ from each of the interval $t_{i-1}<\widehat{t}_{i-1}<t_{i}, i=0,1, \cdots, n$. Let $0=t_{0}^{\prime}<t_{1}^{\prime}<\cdots<t_{2 n}^{\prime}=T$ be the points of subdivision of the refined partition $\pi_{n}^{\prime}$. Define the approximating sum $S_{\pi_{n}^{\prime}}$ as before. We take two steps to prove the assertion in this lemma.

Step 1 . We will first obtain the bounds on $E\left|S_{\pi_{n}}-S_{\pi_{n}^{\prime}}\right|^{2}$.

Let $0 \leq \widetilde{t}_{0}<\widetilde{t}_{1}<\widetilde{t}_{2} \leq T$ be three equally space points on $[0, T]$ and let us denote $X_{\widetilde{t}_{i}}$ by $X_{i}$ and $W_{\tilde{t}_{i}}$ by $W_{i}, i=0,1, \ldots, n$. Define

$$
\begin{aligned}
H & =\frac{f\left(\tilde{t}_{0}, X_{0}\right)}{g\left(\tilde{t}_{0}, X_{0}\right)}\left(W_{2}-W_{0}\right)-\left\{\frac{f\left(\tilde{t}_{1}, X_{1}\right)}{g\left(\tilde{t}_{1}, X_{1}\right)}\left(W_{2}-W_{1}\right)+\frac{f\left(\tilde{t}_{0}, X_{0}\right)}{g\left(\tilde{t}_{0}, X_{0}\right)}\left(W_{1}-W_{0}\right)\right\} \\
& =\left(W_{2}-W_{1}\right)\left\{\frac{f\left(\tilde{t}_{0}, X_{0}\right)}{g\left(\tilde{t}_{0}, X_{0}\right)}-\frac{f\left(\tilde{t}_{1}, X_{1}\right)}{g\left(\tilde{t}_{1}, X_{1}\right)}\right\} .
\end{aligned}
$$

Denote

$$
I=\int_{\tilde{t}_{0}}^{\tilde{t}_{1}} \frac{f\left(t, X_{t}\right)}{g\left(t, X_{t}\right)} d t .
$$

Applying the Taylor expansion, one has

$$
\begin{aligned}
& \frac{f\left(\tilde{t}_{0}, X_{0}\right)}{g\left(\tilde{t}_{0}, X_{0}\right)}-\frac{f\left(\tilde{t}_{1}, X_{1}\right)}{g\left(\tilde{t}_{1}, X_{1}\right)} \\
& =\left(X_{0}-X_{1}\right) \frac{f_{x} g-g_{x} f}{g^{2}}\left(\tilde{t}_{1}, X_{1}\right)+\left(\tilde{t}_{0}-\tilde{t}_{1}\right) \frac{f_{t} g-g_{t} f}{g^{2}}\left(\tilde{t}_{1}, X_{1}\right) \\
& \quad+\frac{1}{2}\left(X_{0}-X_{1}\right)^{2} \frac{\left(f_{x x} g^{2}-g_{x x} f\right) g-2\left(f_{x} g-g_{x} f\right) g g_{x}}{g^{4}}\left(t^{*}, X^{*}\right) \\
& +\frac{1}{2}\left(\tilde{t}_{0}-\tilde{t}_{1}\right)^{2} \frac{\left(f_{t t} g^{2}-g_{t t} f\right) g-2\left(f_{t} g-g_{t} f\right) g g_{t}}{g^{4}}\left(t^{*}, X^{*}\right)
\end{aligned}
$$


Mathematical Problems in Engineering

$$
\begin{aligned}
& +\left(\tilde{t}_{0}-\tilde{t}_{1}\right)\left(X_{0}-X_{1}\right) \frac{\left(f_{t x} g-f g t x-f_{x} g_{t}-f_{t} g_{x}\right) g^{2}+2 f g g_{t} g_{x}}{g^{4}}\left(t^{*}, X^{*}\right) \\
= & -\left(W_{1}-W_{0}+I\right) \frac{f_{x} g-g_{x} f}{g^{2}}\left(\tilde{t}_{1}, X_{1}\right)+\left(\tilde{t}_{0}-\tilde{t}_{1}\right) \frac{f_{t} g-g_{t} f}{g^{2}}\left(\tilde{t}_{1}, X_{1}\right) \\
& +\frac{1}{2}\left(X_{0}-X_{1}\right)^{2} \frac{\left(f_{x x} g^{2}-g_{x x} f\right) g-2\left(f_{x} g-g_{x} f\right) g g_{x}}{g^{4}}\left(t^{*}, X^{*}\right) \\
& +\frac{1}{2}\left(\tilde{t}_{0}-\tilde{t}_{1}\right)^{2} \frac{\left(f_{t t} g^{2}-g_{t t} f\right) g-2\left(f_{t} g-g_{t} f\right) g g_{t}}{g^{4}}\left(t^{*}, X^{*}\right) \\
& +\left(\tilde{t}_{0}-\tilde{t}_{1}\right)\left(X_{0}-X_{1}\right) \frac{\left(f_{t x} g-f g t x-f_{x} g_{t}-f_{t} g_{x}\right) g^{2}+2 f g g_{t} g_{x}}{g^{4}}\left(t^{*}, X^{*}\right),
\end{aligned}
$$

where $\left|X_{1}-X^{*}\right|<\left|X_{0}-X_{1}\right|,\left|\tilde{t}_{1}-t^{*}\right|<\left|\tilde{t}_{0}-\tilde{t}_{1}\right|$.

Relations (3.15) to (3.13) show that

$$
\begin{aligned}
H= & -\left(W_{2}-W_{1}\right) I \frac{f_{x} g-g_{x} f}{g^{2}}\left(\tilde{t}_{1}, X_{1}\right)+\left(W_{2}-W_{1}\right)\left(\tilde{t}_{0}-\tilde{t}_{1}\right) \frac{f_{t} g-g_{t} f}{g^{2}}\left(\tilde{t}_{1}, X_{1}\right) \\
& +\left(W_{2}-W_{1}\right) \frac{1}{2}\left(X_{0}-X_{1}\right)^{2} \frac{\left(f_{x x} g^{2}-g_{x x} f\right) g-2\left(f_{x} g-g_{x} f\right) g g_{x}}{g^{4}}\left(t^{*}, X^{*}\right) \\
& +\left(W_{2}-W_{1}\right) \frac{1}{2}\left(\tilde{t}_{0}-\tilde{t}_{1}\right)^{2} \frac{\left(f_{t t} g^{2}-g_{t t} f\right) g-2\left(f_{t} g-g_{t} f\right) g g_{t}}{g^{4}}\left(t^{*}, X^{*}\right) \\
& +\left(W_{2}-W_{1}\right)\left(\tilde{t}_{0}-\tilde{t}_{1}\right)\left(X_{0}-X_{1}\right) \frac{\left(\mathfrak{f}_{t x} g-f g_{t x}-f_{x} g_{t}-f_{t} g_{x}\right) g^{2}+2 f g g_{t} g_{x}}{g^{4}}\left(t^{*}, X^{*}\right) .
\end{aligned}
$$

Notice that $H^{\prime}$ 's corresponding to different subintervals of $[0, T]$-generated by $\pi_{n}$ form a martingale difference sequence. Observe that

$$
\begin{aligned}
\mathbb{E}|H|^{2}=\mathbb{E}\left(W_{2}-W_{1}\right)^{2} \mathbb{E}\{ & -I \frac{f_{x} g-g_{x} f}{g^{2}}\left(\tilde{t}_{1}, X_{1}\right)+\left(\tilde{t}_{0}-\tilde{t}_{1}\right) \frac{f_{t} g-g_{t} f}{g^{2}}\left(\tilde{t}_{1}, X_{1}\right) \\
& +\frac{1}{2}\left(X_{0}-X_{1}\right)^{2} \frac{\left(f_{x x} g^{2}-g_{x x} f\right) g-2\left(f_{x} g-g_{x} f\right) g g_{x}}{g^{4}}\left(t^{*}, X^{*}\right) \\
& +\frac{1}{2}\left(\tilde{t}_{0}-\tilde{t}_{1}\right)^{2} \frac{\left(f_{t t} g^{2}-g_{t t} f\right) g-2\left(f_{t} g-g_{t} f\right) g g_{t}}{g^{4}}\left(t^{*}, X^{*}\right) \\
& \left.+\left(\tilde{t}_{0}-\tilde{t}_{1}\right)\left(X_{0}-X_{1}\right) \frac{(\mathcal{S}) g^{2}+2 f g g_{t} g_{x}}{g^{4}}\left(t^{*}, X^{*}\right)\right\}^{2}
\end{aligned}
$$


12

Mathematical Problems in Engineering

$$
\begin{aligned}
& \leq 4\left(\tilde{t}_{2}-\tilde{t}_{1}\right)\left\{\mathbb{E}\left(I \frac{f_{x} g-g_{x} f}{g^{2}}\left(\tilde{t}_{1}, X_{1}\right)\right)^{2}+\left(\tilde{t}_{0}-\tilde{t}_{1}\right)^{2} \mathbb{E}\left(\frac{f_{t} g-g_{t} f}{g^{2}}\left(\tilde{t}_{1}, X_{1}\right)\right)^{2}\right. \\
& +\frac{1}{4} \mathbb{E}\left[\left(X_{0}-X_{1}\right)^{4}\left(\frac{\left(f_{x x} g^{2}-g_{x x} f\right) g-2\left(f_{x} g-g_{x} f\right) g g_{x}}{g^{4}}\left(t^{*}, X^{*}\right)\right)^{2}\right] \\
& +\frac{1}{4}\left(\tilde{t}_{0}-\tilde{t}_{1}\right)^{4} \mathbb{E}\left(\frac{\left(f_{t t} g^{2}-g_{t t} f\right) g-2\left(f_{t} g-g_{t} f\right) g g_{t}}{g^{4}}\left(t^{*}, X^{*}\right)\right)^{2} \\
& \left.+\left(\tilde{t}_{0}-\tilde{t}_{1}\right)^{2} \mathbb{E}\left[\left(X_{0}-X_{1}\right)^{2}\left(\frac{(S) g^{2}+2 f g g_{t} g_{x}}{g^{4}}\left(t^{*}, X^{*}\right)\right)^{2}\right]\right\} \\
& \leq 4\left(\tilde{t}_{2}-\tilde{t}_{1}\right)\left\{\left[\mathbb{E} I^{4} \mathbb{E}\left(\frac{f_{x} g-g_{x} f}{g^{2}}\left(\tilde{t}_{1}, X_{1}\right)\right)^{4}\right]^{1 / 2}+\left(\tilde{t}_{0}-\tilde{t}_{1}\right)^{2} \mathbb{E}\left(\frac{f_{t} g-g_{t} f}{g^{2}}\left(\tilde{t}_{1}, X_{1}\right)\right)^{2}\right. \\
& +\frac{1}{4}\left[\mathbb{E}\left(X_{0}-X_{1}\right)^{8} \mathbb{E}\left(\frac{\left(f_{x x} g^{2}-g_{x x} f\right) g-2\left(f_{x} g-g_{x} f\right) g g_{x}}{g^{4}}\left(t^{*}, X^{*}\right)\right)^{8}\right]^{1 / 2} \\
& +\frac{1}{4}\left(\tilde{t}_{0}-\tilde{t}_{1}\right)^{4} \mathbb{E}\left(\frac{\left(f_{t t} g^{2}-g_{t t} f\right) g-2\left(f_{t} g-g_{t} f\right) g g_{t}}{g^{4}}\left(t^{*}, X^{*}\right)\right)^{2} \\
& \left.+\left(\tilde{t}_{0}-\tilde{t}_{1}\right)^{2}\left[\mathbb{E}\left(X_{0}-X_{1}\right)^{4} \mathbb{E}\left(\frac{(\mathcal{S}) g^{2}+2 f g g_{t} g_{x}}{g^{4}}\left(t^{*}, X^{*}\right)\right)^{4}\right]^{1 / 2}\right\} \\
& \leq 4\left(\tilde{t}_{2}-\tilde{t}_{1}\right)\left\{\left(\mathbb{E} I^{4}\right)^{1 / 2} C\left[\left(\mathbb{E} f_{x}^{8}\left(\tilde{t}_{1}, X_{1}\right)\right)^{1 / 2}+\left(\mathbb{E} g_{x}^{8}\left(\tilde{t}_{1}, X_{1}\right)\right)^{1 / 2}+\left(\mathbb{E} f^{8}\left(\tilde{t}_{1}, X_{1}\right)\right)^{1 / 2}\right]^{1 / 2}\right. \\
& +\left(\tilde{t}_{0}-\tilde{t}_{1}\right)^{2} C\left[\left(\mathbb{E} f_{t}^{4}\left(\tilde{t}_{1}, X_{1}\right)\right)^{1 / 2}+\left(\mathbb{E} g_{t}^{4}\left(\tilde{t}_{1}, X_{1}\right)\right)^{1 / 2}+\left(\mathbb{E} f^{4}\left(\tilde{t}_{1}, X_{1}\right)\right)^{1 / 2}\right] \\
& +\frac{1}{4}\left(\mathbb{E}\left(X_{0}-X_{1}\right)^{8}\right)^{1 / 2} C\left[\left(\mathbb{E} f_{x x}^{8}\left(t^{*}, X^{*}\right)\right)^{1 / 2}+\left(\mathbb{E} g_{x x}^{8}\left(t^{*}, X^{*}\right)\right)^{1 / 2}\right. \\
& +\left(\mathbb{E} f^{8}\left(t^{*}, X^{*}\right)\right)^{1 / 2}+\left(\mathbb{E} f_{x}^{8}\left(t^{*}, X^{*}\right)\right)^{1 / 2} \\
& \left.+\left(\mathbb{E} f_{x}^{16}\left(t^{*}, X^{*}\right)\right)^{1 / 2}\right]^{1 / 2} \\
& +\frac{1}{4}\left(\tilde{t}_{0}-\tilde{t}_{1}\right)^{4} C\left[\left(\mathbb{E} f_{t t}^{4}\left(t^{*}, X^{*}\right)\right)^{1 / 2}+\left(\mathbb{E} g_{t t}^{4}\left(t^{*}, X^{*}\right)\right)^{1 / 2}+\left(\mathbb{E} f_{t}^{4}\left(t^{*}, X^{*}\right)\right)^{1 / 2}\right. \\
& \left.+\left(\mathbb{E} g_{t}^{4}\left(t^{*}, X^{*}\right)\right)^{1 / 2}+\left(\mathbb{E} g_{t}^{8}\left(t^{*}, X^{*}\right)\right)^{1 / 2}\right]
\end{aligned}
$$


Mathematical Problems in Engineering

$$
\begin{aligned}
+\left(\tilde{t}_{0}-\tilde{t}_{1}\right)^{2}\left[\mathbb{E}\left(X_{0}-X_{1}\right)^{4}\right]^{1 / 2}[ & \left(\mathbb{E} f_{t x}^{8}\left(t^{*}, X^{*}\right)\right)^{1 / 2}+\left(\mathbb{E} g_{t x}^{8}\left(t^{*}, X^{*}\right)\right)^{1 / 2} \\
& +\left(\mathbb{E} f_{x}^{8}\left(\tilde{t}_{1}, X_{1}\right)\right)^{1 / 2}+\left(\mathbb{E} g_{x}^{8}\left(\tilde{t}_{1}, X_{1}\right)\right)^{1 / 2} \\
& \left.\left.+\left(\mathbb{E} f_{t}^{8}\left(\tilde{t}_{1}, X_{1}\right)\right)^{1 / 2}+\left(\mathbb{E} g_{t}^{8}\left(\tilde{t}_{1}, X_{1}\right)\right)^{1 / 2}\right]^{1 / 2}\right\} .
\end{aligned}
$$

where $\mathcal{S}$ denotes $f_{t x} g-f g_{t x}-f_{x} g_{t}-f_{t} g_{x}$.

By Theorem 4 of [31], for any $0 \leq s<t \leq T$, there exists $C>0$ such that

$$
\mathbb{E}\left(X_{t}-X_{s}\right)^{2 m} \leq C\left(\mathbb{E} X_{0}^{2 m}+1\right)(t-s)^{m}, \quad m \geq 1 .
$$

Hence

$$
\begin{aligned}
& \mathbb{E}\left(X_{t}-X_{s}\right)^{8} \leq C\left(\mathbb{E} X_{0}^{8}+1\right)(t-s)^{4}, \\
& \mathbb{E}\left(X_{t}-X_{s}\right)^{4} \leq C\left(\mathbb{E} X_{0}^{4}+1\right)(t-s)^{2} .
\end{aligned}
$$

Furthermore by (A2) and (A3), we have

$$
\begin{aligned}
\mathbb{E} I^{4} & =\mathbb{E}\left(\int_{\tilde{t}_{0}}^{\tilde{t}_{1}} \frac{f\left(t, X_{t}\right)}{g\left(t, X_{t}\right)} d t\right)^{4} \\
& \leq C \mathbb{E}\left(\int_{\tilde{t}_{0}}^{\tilde{t}_{1}} f^{4}\left(t, X_{t}\right) d t\right) \\
& \leq C \mathbb{E}\left(\int_{\tilde{t}_{0}}^{\tilde{t}_{1}}\left(1+\left|X_{t}\right|^{2}\right)^{2} d t\right) \\
& \leq C\left(\tilde{t}_{1}-\tilde{t}_{0}\right)^{4} \sup _{0 \leq t \leq T} \mathbb{E}\left(1+\left|X_{t}\right|^{2}\right)^{2} \\
& \leq C\left(\tilde{t}_{1}-\tilde{t}_{0}\right)^{4} .
\end{aligned}
$$

Thus

$$
\mathbb{E}(H)^{2} \leq C\left(\tilde{t}_{2}-\tilde{t}_{1}\right)\left(\tilde{t}_{1}-\tilde{t}_{0}\right)^{2}
$$


Using the property that $H$ corresponding to different subintervals forms a martingale difference sequence, it follows that

$$
\mathbb{E}\left|S_{\pi_{n}}-S_{\pi_{n}^{\prime}}\right|^{2} \leq C \frac{T^{3}}{n^{2}}
$$

for some constant $C>0$.

Step 2. We will show now the bounds on $E\left|S_{\pi_{n}^{\prime}}-S\right|^{2}$.

Let $\pi_{n}^{(p)}, p \geq 0$ be the sequence of partitions such that $\pi_{n}^{(i+1)}$ is a refinement of $\pi_{n}^{(n)}$ by choosing the midpoint of the subintervals generated by $\pi_{n}^{(n)}$. Note that $\pi_{n}^{(0)}=\pi_{n}$ and $\pi_{n}^{(1)}=\pi_{n}^{\prime}$. The analysis given above proves that

$$
\mathbb{E}\left|S_{\pi_{n}}(p)-S_{\pi_{n}(p+1)}\right|^{2} \leq C \frac{T^{3}}{2^{p} n^{2}}, \quad p \geq 0,
$$

where $S_{\pi_{n}}(p)$ is the approximation corresponding to $\pi_{n}^{(p)}$ and $S_{\pi_{n}}(0)=S_{\pi_{n}}$.

Therefore, applying the Hölder inequality and the Minkovski inequality, one gets

$$
\begin{aligned}
\mathbb{E}\left|S_{\pi_{n}}(0)-S_{\pi_{n}(p+1)}\right|^{2} & \leq \mathbb{E}\left[\sum_{k=0}^{p}\left(S_{\pi_{n}}(k)-S_{\pi_{n}(k+1)}\right)\right]^{2} \\
\leq & {\left[\sum_{k=0}^{p}\left(\mathbb{E}\left|S_{\pi_{n}}(k)-S_{\pi_{n}(k+1)}\right|^{2}\right)^{1 / 2}\right]^{2} } \\
& \leq\left[\sum_{k=0}^{p}\left(\frac{C T^{3}}{2^{p} n^{2}}\right)^{1 / 2}\right]^{2} \\
& \leq C \frac{T^{3}}{n^{2}}
\end{aligned}
$$

for all $p \geq 0$. Let $p \rightarrow \infty$. Since the integral $S$ exists, $S_{\pi_{n}}(p+1)$ converges in $\mathcal{L}_{2}$ to $S$ as $p \rightarrow \infty$. Note that $\pi_{n}^{(p+1)}, P \geq 0$ is a sequence of partitions such that the mesh of the partition tends to zero as $p \rightarrow \infty$ for any fixed $n$.

Thus

$$
\mathbb{E}\left|S_{\pi_{n}}-S\right|^{2} \leq C \frac{T^{3}}{2^{p} n^{2}}, \quad p \geq 0,
$$


where

$$
S=\lim _{n \rightarrow \infty} S_{\pi_{n}}=\int_{0}^{T} \frac{f\left(t, X_{t}\right)}{g\left(t, X_{t}\right)} d W_{t}
$$

The proof is now complete.

Theorem 3.3. Under assumptions $(A 1)-(A 4),(A 5)_{2},(A 6)_{2},(A 7)$, and (A8), one has

$$
\begin{aligned}
& \mathbb{E}\left|L_{n, T}(\theta)-L_{T}(\theta)\right|^{2} \leq C \frac{T^{3}}{n^{2}}, \\
& \mathbb{E}\left|L_{n, T}^{\prime}(\theta)-L_{T}^{\prime}(\theta)\right|^{2} \leq C \frac{T^{3}}{n^{2}} .
\end{aligned}
$$

Proof. By the analysis given above, one has

$$
\begin{aligned}
&\left|L_{n, T}(\theta)-L_{T}(\theta)\right|^{2} \\
&=\mid \theta\left[\sum_{i=1}^{n} \frac{f\left(t_{i-1}, X_{t_{i-1}}\right)}{g^{2}\left(t_{i-1}, X_{t_{i-1}}\right)}\left(X_{t_{i}}-X_{t_{i-1}}\right)-\int_{0}^{T} \frac{f\left(t, X_{t}\right)}{g^{2}\left(t, X_{t}\right)} d X_{t}\right] \\
& \quad-\left.\frac{\theta^{2}}{2}\left[\sum_{i=1}^{n} \frac{f^{2}\left(t_{i-1}, X_{t_{i-1}}\right)}{g^{2}\left(t_{i-1}, X_{t_{i-1}}\right)}\left(t_{i}-t_{i-1}\right)-\int_{0}^{T} \frac{f^{2}\left(t, X_{t}\right)}{g^{2}\left(t, X_{t}\right)} d t\right]\right|^{2} \\
& \leq \frac{\theta^{4}}{2}\left|\sum_{i=1}^{n} \frac{f^{2}\left(t_{i-1}, X_{t_{i-1}}\right)}{g^{2}\left(t_{i-1}, X_{t_{i-1}}\right)}\left(t_{i}-t_{i-1}\right)-\int_{0}^{T} \frac{f^{2}\left(t, X_{t}\right)}{g^{2}\left(t, X_{t}\right)} d t\right|^{2} \\
& \quad+2 \theta^{2}\left|\sum_{i=1}^{n} \frac{f\left(t_{i-1}, X_{t_{i-1}}\right)}{g^{2}\left(t_{i-1}, X_{t_{i-1}}\right)}\left(W_{t_{i}}-W_{t_{i-1}}\right)-\int_{0}^{T} \frac{f\left(t, X_{t}\right)}{\mathrm{g}^{2}\left(t, X_{t}\right)} d W_{t}\right|^{2} .
\end{aligned}
$$

Hence, it follows from Lemmas 3.1 and 3.2 that

$$
\begin{aligned}
& \mathbb{E}\left|L_{n, T}(\theta)-L_{T}(\theta)\right|^{2} \\
& \leq \frac{\theta^{4}}{2} \mathbb{E}\left|\sum_{i=1}^{n} \frac{f^{2}\left(t_{i-1}, X_{t_{i-1}}\right)}{g^{2}\left(t_{i-1}, X_{t_{i-1}}\right)}\left(t_{i}-t_{i-1}\right)-\int_{0}^{T} \frac{f^{2}\left(t, X_{t}\right)}{g^{2}\left(t, X_{t}\right)} d t\right|^{2} \\
& \quad+2 \theta^{2} \mathbb{E}\left|\sum_{i=1}^{n} \frac{f\left(t_{i-1}, X_{t_{i-1}}\right)}{g^{2}\left(t_{i-1}, X_{t_{i-1}}\right)}\left(W_{t_{i}}-W_{t_{i-1}}\right)-\int_{0}^{T} \frac{f\left(t, X_{t}\right)}{g^{2}\left(t, X_{t}\right)} d W_{t}\right|^{2} \\
& \leq C \frac{T^{3}}{n^{2}} .
\end{aligned}
$$


Next, note that

$$
\begin{aligned}
\left|L_{n, T}^{\prime}(\theta)-L_{T}^{\prime}(\theta)\right|^{2} & \mid \theta \sum_{i=1}^{n} \frac{f\left(t_{i-1}, X_{t_{i-1}}\right)}{g^{2}\left(t_{i-1}, X_{t_{i-1}}\right)}\left(X_{t_{i}}-X_{t_{i-1}}\right) \\
= & -\theta \sum_{i=1}^{n} \frac{f^{2}\left(t_{i-1}, X_{t_{i-1}}\right)}{g^{2}\left(t_{i-1}, X_{t_{i-1}}\right)}\left(t_{i}-t_{i-1}\right)-\left.\left[\int_{0}^{T} \frac{f\left(t, X_{t}\right)}{g^{2}\left(t, X_{t}\right)} d X_{t}-\int_{0}^{T} \frac{f^{2}\left(t, X_{t}\right)}{g^{2}\left(t, X_{t}\right)} d t\right]\right|^{2} \\
= & (1-\theta)^{2}\left|\sum_{i=1}^{n} \frac{f^{2}\left(t_{i-1}, X_{t_{i-1}}\right)}{g^{2}\left(t_{i-1}, X_{t_{i-1}}\right)}\left(t_{i}-t_{i-1}\right)-\int_{0}^{T} \frac{f^{2}\left(t, X_{t}\right)}{g^{2}\left(t, X_{t}\right)} d t\right|^{2} \\
& +\left|\sum_{i=1}^{n} \frac{f\left(t_{i-1}, X_{t_{i-1}}\right)}{g^{2}\left(t_{i-1}, X_{t_{i-1}}\right)}\left(W_{t_{i}}-W_{t_{i-1}}\right)-\int_{0}^{T} \frac{f\left(t, X_{t}\right)}{g^{2}\left(t, X_{t}\right)} d W_{t}\right|^{2} .
\end{aligned}
$$

Similarly, by Lemmas 3.1 and 3.2, we obtain

$$
\mathbb{E}\left|L_{n, T}^{\prime}(\theta)-L_{T}^{\prime}(\theta)\right|^{2} \leq C \frac{T^{3}}{n^{2}}
$$

The proof is now complete.

Remark 3.4. The rate of convergence of the approximations for Itô and ordinary integral have been investigated in Lemmas 3.1 and 3.2. Based on these analysis results, the rate of convergence of the approximate log-likelihood function for nonlinear nonhomogenous stochastic system with unknown parameter has been established in Theorem 3.3. It should be pointed out that the corresponding approximate result gained in [27] is the special case for linear stochastic differential equation, furthermore, the conclusions in [9] also can be regarded as a special example under the result in Theorem 3.3 for nonlinear nonhomogenous stochastic system with constant diffusion.

Finally, we will study the error bound in probability between the AMLE and the continuous MLE for nonlinear nonhomogenous stochastic system with unknown parameter.

Theorem 3.5. Under assumption $(A 1)-(A 4),(A 5)_{2},(A 6)_{2},(A 7)$, and $(A 8)$, one has

$$
\mathbb{E}\left|\theta_{n, T}-\theta_{T}\right|^{2} \leq C \frac{T^{3}}{n^{2}} .
$$

Proof. We know $\theta_{n, T}$ and $\theta_{T}$ are the solutions of equations $L_{n, T}^{\prime}(\theta)=0$ and $L_{T}^{\prime}(\theta)=0$, respectively. 
Hence, one gets

$$
\begin{aligned}
\mid \theta_{n, T}- & \left.\theta_{T}\right|^{2} \\
= & \left|\frac{\sum_{i=1}^{n}\left(f\left(t_{i-1}, X_{t_{i-1}}\right) / g^{2}\left(t_{i-1}, X_{t_{i-1}}\right)\right)\left(X_{t_{i}}-X_{t_{i-1}}\right)}{\sum_{i=1}^{n}\left(f^{2}\left(t_{i-1}, X_{t_{i-1}}\right) / g^{2}\left(t_{i-1}, X_{t_{i-1}}\right)\right)\left(t_{i}-t_{i-1}\right)}-\frac{\int_{0}^{T}\left(f\left(t, X_{t}\right) / g^{2}\left(t, X_{t}\right)\right) d X_{t}}{\int_{0}^{T}\left(f^{2}\left(t, X_{t}\right) / g^{2}\left(t, X_{t}\right)\right) d t}\right|^{2} \\
= & \left|\frac{\sum_{i=1}^{n}\left(f\left(t_{i-1}, X_{t_{i-1}}\right) / g\left(t_{i-1}, X_{t_{i-1}}\right)\right)\left(W_{t_{i}}-W_{t_{i-1}}\right)}{\sum_{i=1}^{n}\left(f^{2}\left(t_{i-1}, X_{t_{i-1}}\right) / g^{2}\left(t_{i-1}, X_{t_{i-1}}\right)\right)\left(t_{i}-t_{i-1}\right)}-\frac{\int_{0}^{T}\left(f\left(t, X_{t}\right) / g\left(t, X_{t}\right)\right) d W_{t}}{\int_{0}^{T}\left(f^{2}\left(t, X_{t}\right) / g^{2}\left(t, X_{t}\right)\right) d t}\right|^{2} \\
= & \mid \frac{\sum_{i=1}^{n}\left(f\left(t_{i-1}, X_{t_{i-1}}\right) / g\left(t_{i-1}, X_{t_{i-1}}\right)\right)\left(W_{t_{i}}-W_{t_{i-1}}\right)-\int_{0}^{T}\left(f\left(t, X_{t}\right) / g\left(t, X_{t}\right)\right) d W_{t}}{\sum_{i=1}^{n}\left(f^{2}\left(t_{i-1}, X_{t_{i-1}}\right) / g^{2}\left(t_{i-1}, X_{t_{i-1}}\right)\right)\left(t_{i}-t_{i-1}\right)} \\
& -\left.\frac{\int_{0}^{T}\left(f\left(t, X_{t}\right) / g\left(t, X_{t}\right)\right) d W_{t}\left[\sum_{i=1}^{n}\left(f^{2}\left(t_{i-1}, X_{t_{i-1}}\right) / g^{2}\left(t_{i-1}, X_{t_{i-1}}\right)\right)\left(t_{i}-t_{i-1}\right)-\mathcal{A}\right]}{\left[\sum_{i=1}^{n}\left(f^{2}\left(t_{i-1}, X_{t_{i-1}}\right) / g^{2}\left(t_{i-1}, X_{t_{i-1}}\right)\right)\left(t_{i}-t_{i-1}\right)\right] \int_{0}^{T}\left(f^{2}\left(t, X_{t}\right) / g^{2}\left(t, X_{t}\right)\right) d t}\right|^{2} \\
\leq & 2\left|\frac{\sum_{i=1}^{n}\left(f\left(t_{i-1}, X_{t_{i-1}}\right) / g\left(t_{i-1}, X_{t_{i-1}}\right)\right)\left(W_{t_{i}}-W_{t_{i-1}}\right)-\int_{0}^{T}\left(f\left(t, X_{t}\right) / g\left(t, X_{t}\right)\right) d W_{t}}{\sum_{i=1}^{n}\left(f^{2}\left(t_{i-1}, X_{t_{i-1}}\right) / g^{2}\left(t_{i-1}, X_{t_{i-1}}\right)\right)\left(t_{i}-t_{i-1}\right)}\right|^{2} \\
& +2\left|\frac{\int_{0}^{T}\left(f\left(t, X_{t}\right) / g\left(t, X_{t}\right)\right) d W_{t}\left[\sum_{i=1}^{n}\left(f^{2}\left(t_{i-1}, X_{t_{i-1}}\right) / g^{2}\left(t_{i-1}, X_{t_{i-1}}\right)\right)\left(t_{i}-t_{i-1}\right)-\mathscr{A}\right]}{\left[\sum_{i=1}^{n}\left(f^{2}\left(t_{i-1}, X_{t_{i-1}}\right) / g^{2}\left(t_{i-1}, X_{t_{i-1}}\right)\right)\left(t_{i}-t_{i-1}\right)\right] \int_{0}^{T}\left(f^{2}\left(t, X_{t}\right) / g^{2}\left(t, X_{t}\right)\right) d t}\right|^{2} .
\end{aligned}
$$

As we know that $\sum_{i=1}^{n}\left(f^{2}\left(t_{i-1}, X_{t_{i-1}}\right) / g^{2}\left(t_{i-1}, X_{t_{i-1}}\right)\right)\left(t_{i}-t_{i-1}\right)>0$, so there exists a constant $C>0$ such that

$$
\frac{1}{\sum_{i=1}^{n}\left(f^{2}\left(t_{i-1}, X_{t_{i-1}}\right) / g^{2}\left(t_{i-1}, X_{t_{i-1}}\right)\right)\left(t_{i}-t_{i-1}\right)} \leq C
$$

Therefore, applying Itô isomorphism, the Cauchy-Schwarz inequality, Lemmas 3.1 and 3.2, we obtain

$$
\begin{aligned}
\mathbb{E}\left|\theta_{n, T}-\theta_{T}\right|^{2} & \\
\leq & 2 \mathbb{E}\left|\frac{\sum_{i=1}^{n}\left(f\left(t_{i-1}, X_{t_{i-1}}\right) / g\left(t_{i-1}, X_{t_{i-1}}\right)\right)\left(W_{t_{i}}-W_{t_{i-1}}\right)-\int_{0}^{T}\left(f\left(t, X_{t}\right) / g\left(t, X_{t}\right)\right) d W_{t}}{\sum_{i=1}^{n}\left(f^{2}\left(t_{i-1}, X_{t_{i-1}}\right) / g^{2}\left(t_{i-1}, X_{t_{i-1}}\right)\right)\left(t_{i}-t_{i-1}\right)}\right|^{2} \\
& +2 \mathbb{E}\left|\frac{\int_{0}^{T}\left(f\left(t, X_{t}\right) / g\left(t, X_{t}\right)\right) d W_{t}\left[\sum_{i=1}^{n}\left(f^{2}\left(t_{i-1}, X_{t_{i-1}}\right) / g^{2}\left(t_{i-1}, X_{t_{i-1}}\right)\right)\left(t_{i}-t_{i-1}\right)-\mathcal{A}\right]}{\left[\sum_{i=1}^{n}\left(f^{2}\left(t_{i-1}, X_{t_{i-1}}\right) / g^{2}\left(t_{i-1}, X_{t_{i-1}}\right)\right)\left(t_{i}-t_{i-1}\right)\right] \int_{0}^{T}\left(f^{2}\left(t, X_{t}\right) / g^{2}\left(t, X_{t}\right)\right) d t}\right|^{2}
\end{aligned}
$$




$$
\begin{aligned}
\leq & C \mathbb{E}\left|\sum_{i=1}^{n}\left(f\left(t_{i-1}, X_{t_{i-1}}\right) / g\left(t_{i-1}, X_{t_{i-1}}\right)\right)\left(W_{t_{i}}-W_{t_{i-1}}\right)-\int_{0}^{T}\left(f\left(t, X_{t}\right) / g\left(t, X_{t}\right)\right) d W_{t}\right|^{2} \\
& +C \mathbb{E}\left|\sum_{i=1}^{n}\left(f^{2}\left(t_{i-1}, X_{t_{i-1}}\right) / g^{2}\left(t_{i-1}, X_{t_{i-1}}\right)\right)\left(t_{i}-t_{i-1}\right)-\int_{0}^{T}\left(f^{2}\left(t, X_{t}\right) / g^{2}\left(t, X_{t}\right)\right) d t\right|^{2} \\
\leq & C \frac{T^{3}}{n^{2}}
\end{aligned}
$$

where $A$ denotes $\int_{0}^{T}\left(f^{2}\left(t, X_{t}\right) / g^{2}\left(t, X_{t}\right)\right) d t$.

The proof is now complete.

Remark 3.6. Up to present, the rate of the convergence of the approximate log-likelihood functions and the error bound in probability between the AMLE and the continuous MLE have been obtained for the nonlinear nonhomogenous stochastic system with unknown parameter. As well, the corresponding results gained in $[9,27]$ are the direct conclusions after applying Chebyshev's inequality on (3.32).

\section{Conclusions}

In this paper, we have investigated the error bound in probability between the ALME and the continuous MLE for a class of general nonlinear nonhomogenous stochastic system with unknown parameter. The rates of convergence of the approximations for Itô and ordinary integral have been derived under some regular assumptions. On the basis of these analysis results, we have studied the in probability rate of convergence of the approximate log-likelihood function to the true continuous log-likelihood function for the nonlinear nonhomogenous stochastic system involving unknown parameter. Finally, the main result which gives the error bound in probability between the ALME and the continuous MLE has been established. It should be noted that one of the future research topics would be to investigate the asymptotic normality of the ALME for the nonlinear nonhomogenous stochastic system with unknown parameter mentioned in this paper.

\section{Acknowledgment}

This work was supported in part by the National Natural Science Foundation of China under Grant 60974030.

\section{References}

[1] M. Arato, A. N. Kolmogrorov, and Y. G. Sinai, "On parameter estimation of a complex stationary gaussian process," Doklady of Academy of Sciences, vol. 146, no. 4, pp. 747-750, 1962.

[2] H. Dong, Z. Wang, and H. Gao, " $H_{\infty}$ fuzzy control for systems with repeated scalar nonlinearities and random packet losses," IEEE Transactions on Fuzzy Systems, vol. 17, no. 2, pp. 440-450, 2009.

[3] H. Dong, Z. Wang, and H. Gao, "Robust $H_{\infty}$ filtering for a class of nonlinear networked systems with multiple stochastic communication delays and packet dropouts," IEEE Transactions on Signal Processing, vol. 58, no. 4, pp. 1957-1966, 2010. 
[4] H. Dong, Z. Wang, and H. Gao, "Distributed filtering for a class of time-varying systems over sensor networks with quantizationerrors and successive packet dropouts," IEEE Transactions on Signal Processing, vol. 60, no. 6, pp. 3164-3173, 2012.

[5] W. B. Michael and S. C. Pedro, "Simulated likelihood estimation of diffusions with an application to exchange rate dynamics inincomplete markets," Journal of Financial Economics, vol. 63, no. 2, pp. 161-210, 2002.

[6] F. Konecny, "Maximum likelihood estimation of a drift parameter from a partially observed diffusion in the case of small measurement noise," Statistics \& Decisions, vol. 8, no. 2, pp. 115-130, 1990.

[7] B. L. S. Prakasa Rao, "On Bayes estimation for diffusion fields," in Statistics: Applications and New Directions, Proceedings of the ISI Golden Jubilee Conferences, J. K. Ghosh and J. Roy, Eds., pp. 504-511, Indian Statistical Institute, Calcutta, India, 1981.

[8] P. J. Huber, "Robust estimation of a location parameter," Annals of Mathematical Statistics, vol. 35, pp. 73-101, 1964.

[9] J. P. N. Bishwal, Prameter Estimation in Stochastic Differential Equations, vol. 1923 of Lecture Notes in Mathematics, Springer, Berlin, Germany, 2008.

[10] V. Genon-Catalot, "Maximum contrast estimation for diffusion processes from discrete observations," Statistics, vol. 21, no. 1, pp. 99-116, 1990.

[11] J. Hu, Z. Wang, H. Gao, and L. K. Stergioulas, "Robust $H_{\infty}$ sliding mode control for discrete timedelay systems with stochastic nonlinearities," Journal of the Franklin Institute, vol. 349, no. 4, pp. 14591479, 2012.

[12] J. Hu, Z. Wang, H. Gao, and L. K. Stergioulas, "Probability-guaranteed $H_{\infty}$ finite-horizon filtering for a class of nonlinear timevaryingsystems with sensor saturation," System and Control Letters, vol. 61, no. 4, pp. 477-484, 2012.

[13] J. Hu, Z. Wang, G. Niu, and L. K. Stergioulas, " $H_{\infty}$ sliding mode observer design for a class of nonlinear discrete time-delaysystems: a delay-fractioningapproach," International Journal of Robust and Nonlinear Control. In press.

[14] V. Lánská, "Minimum contrast estimation in diffusion processes," Journal of Applied Probability, vol. 16, no. 1, pp. 65-75, 1979 .

[15] L. Birgé and P. Massart, "Rates of convergence for minimum contrast estimators," Probability Theory and Related Fields, vol. 97, no. 1-2, pp. 113-150, 1993.

[16] B. Shen, Z. Wang, H. Shu, and G. Wei, “On nonlinear $H_{\infty}$ filtering for discrete-time stochastic systems with missing measurements," IEEE Transactions on Automatic Control, vol. 53, no. 9, pp. 2170-2180, 2008.

[17] B. Shen, Z. Wang, Y. S. Hung, and G. Chesi, "Distributed $H_{\infty}$ filtering for polynomial nonlinear stochastic systems in sensor networks," IEEE Transactions on Industrial Electronics, vol. 58, no. 5, pp. 1971-1979, 2011.

[18] B. Shen, Z. Wang, and X. Liu, "A stochastic sampled-data approach to distributed $H_{\infty}$ filtering in sensor networks," IEEE Transactions on Circuits and Systems I, vol. 58, no. 9, pp. 2237-2246, 2011.

[19] V. Harison, "The Bernstein-von Mises theorem for a certain class of Gaussion diffusion processes," Publications du Service deMathematiques, Umiversite d'Antananarivo, vol. 6, pp. 1-7, 1992.

[20] D. Levanony, A. Shwartz, and O. Zeitouni, "Recursive identification in continuous-time stochastic processes," Stochastic Processes and Their Applications, vol. 49, no. 2, pp. 245-275, 1994.

[21] Ju. A. Kutojanc, "Estimation of a parameter of a diffusion type process," Akademiya Nauk SSSR. Teoriya Veroyatnosteŭ i ee Primeneniya, vol. 23, no. 3, pp. 665-672, 1978.

[22] V. Borkar and A. Bagchi, "Parameter estimation in continuous-time stochastic processes," Stochastics, vol. 8, no. 3, pp. 193-212, 1982.

[23] J. Hu, Z. Wang, and H. Gao, "A delay fractioning approach to robust sliding mode control for discretetime stochastic systems with randomly occurring non-linearities," IMA Journal of Mathematical Control and Information, vol. 28, no. 3, pp. 345-363, 2011.

[24] J. Hu, Z. Wang, H. Gao, and L. K. Stergioulas, "Robust sliding mode control for discrete stochastic systems with mixed timedelays, randomly occurring uncertainties and randomly occurring nonlinearities," IEEE Transactions on Industrial Electronics, vol. 59, no. 7, pp. 3008-3015, 2012.

[25] D. Dacunha-Castelle and D. Florens-Zmirou, "Estimation of the coefficients of a diffusion from discrete observations," Stochastics, vol. 19, no. 4, pp. 263-284, 1986.

[26] B. L. S. Prakasa Rao, Statistical Inference for Diffusion Type Processes, vol. 8 of Kendall's Library of Statistics, Edward Arnold, Oxford University Press, London, UK, 1999.

[27] A. Le Breton, "On continuous and discrete sampling for parameter estimation in diffusion type processes," Mathematical Programming Study, vol. 5, pp. 124-144, 1976. 
[28] A. R. Pedersen, "Consistency and asymptotic normality of an approximate maximum likelihood estimator for discretely observed diffusion processes," Bernoulli, vol. 1, no. 3, pp. 257-279, 1995.

[29] Y. Ait-Sahalia, "Maximum likelihood estimation of discretely sampled diffusions: a closed-form approximation approach," Econometrica, vol. 70, no. 1, pp. 223-262, 2002.

[30] X. Mao, Stochastic Differential Equations and Their Applications, Horwood Publishing Series in Mathematics \& Applications, Horwood Publishing, Chichester, UK, 1997.

[31] I. I. Gikham and A. V. Skorohod, Stochastic Differential Equations, Springer, Berlin, Germany, 1972. 


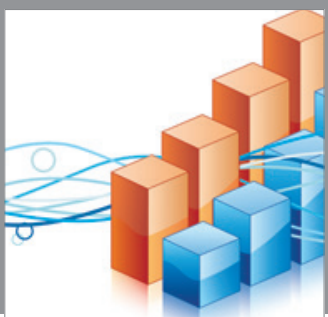

Advances in

Operations Research

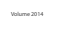

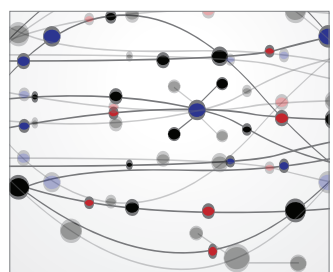

\section{The Scientific} World Journal
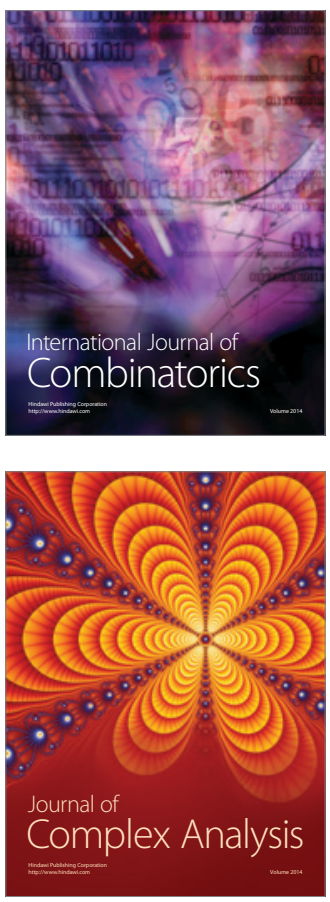

International Journal of

Mathematics and

Mathematical

Sciences
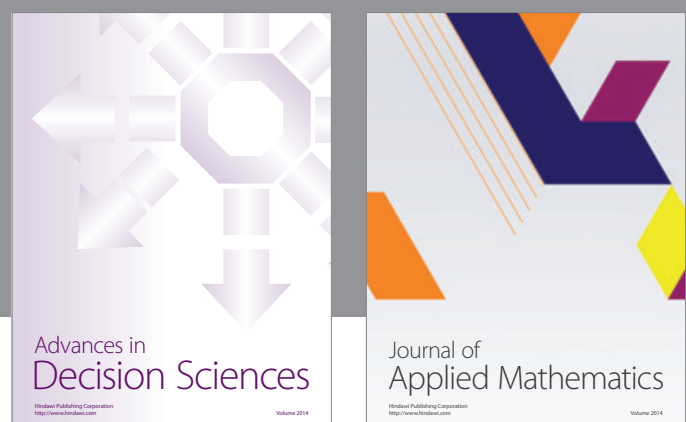

Journal of

Applied Mathematics
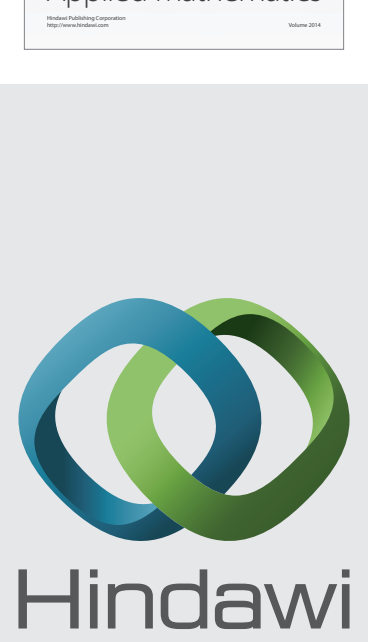

Submit your manuscripts at http://www.hindawi.com
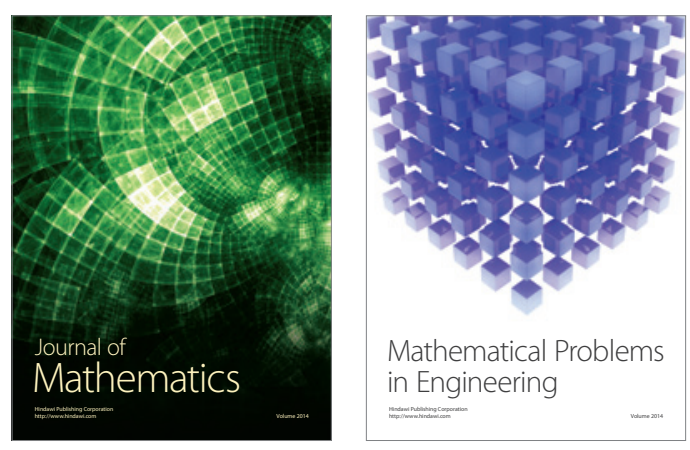

Mathematical Problems in Engineering
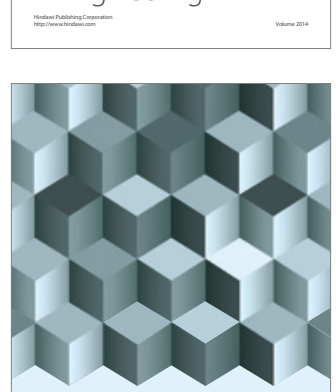

Journal of

Function Spaces


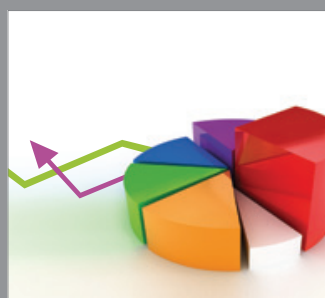

ournal of

Probability and Statistics

Promensencen
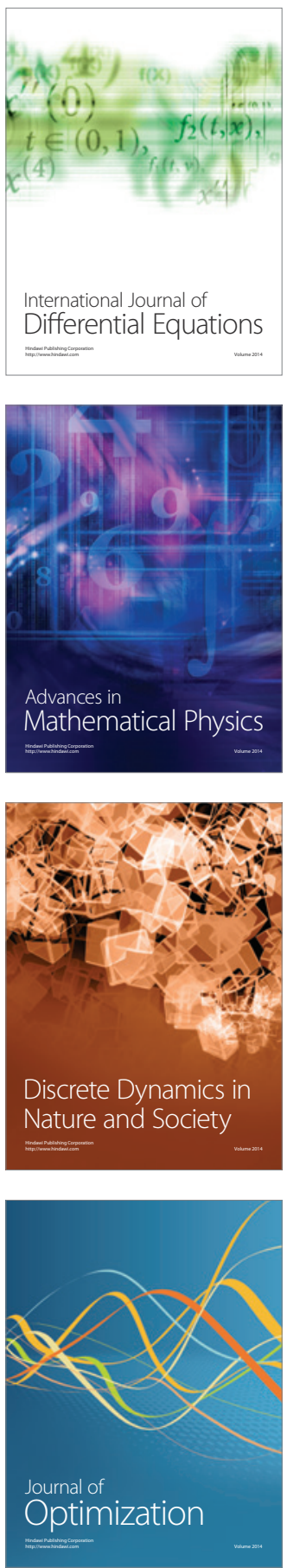